\title{
SIGNATURES AND POSSIBLE EVIDENCE FOR SUPERSYMMETRY AT THE CERN COLLIDER
}

\author{
Howard E. HABER ${ }^{1}$ \\ Department of Physics, University of Califomia, Santa Cruz, CA 95064, USA \\ and \\ G.L. KANE ${ }^{2}$ \\ Randall Laboratory of Physics, University of Michigan, Ann Arbor, MI 48109, USA
}

Received 9 April 1984

\begin{abstract}
For certain ranges of masses of the supersymmetric partners of the gluons, quarks and $W$-bosons, it is expected that a few events of production and decay of the partners could be observed at the CERN p $\bar{p}$ collider. The characteristic signa-

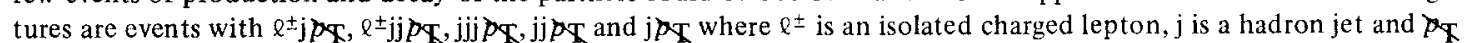
stands for missing $p_{\mathrm{T}}$. Some recently reported events are of this type.
\end{abstract}

Although supersymmetry has been studied extensively for over a decade, both as a quantum field theory and as a possible symmetry of nature, so far there has been no hint of experimental evidence for supersymmetry ${ }^{\ddagger 1}$. Recently some events have been reported by the UA2 group at the CERN $\bar{p}$ p collider [5] which have the characteristics expected for the production and decay of supersymmetric partners of gauge bosons and quarks (for certain restricted ranges of masses for the particles in question). In this note, wc will explain the signatures and rates for these events, and emphasize some predictions which must hold if the events could be interpreted as evidence for supersymmetry.

In a supersymmetric theory the standard particles, quarks (q), gluons $(\mathrm{g})$, weak gauge bosons $\left(\mathrm{W}^{ \pm}, \mathrm{Z}^{0}\right)$, leptons $\left(\ell^{ \pm}, v\right)$, photon $(\gamma)$ and Higgs bosons $(H)$ have

1 Supported in part by the National Science Foundation, grant PHY-81 15541.

2 Supported in part by the Department of Energy, Contract DE-AC02-76ER01112.

$\neq 1$ Reviews of supersymmetry as a quantum field theory are given in ref. [1], reviews of supersymmetric model-building are given in ref. [2], and reviews of supersymmetric phenomenology are given in refs. $[3,4]$. partners differing by $1 / 2$ unit in spin but identical in all other quantum numbers, scalar quarks $(\tilde{\mathrm{q}})$, gluinos $(\widetilde{\mathrm{g}})$, winos $(\widetilde{\mathrm{W}}), \operatorname{zinos}(\widetilde{\mathrm{z}})$, scalar leptons $\left(\tilde{\mathrm{Q}}^{ \pm}\right)$, scalar neutrinos $(\tilde{v})$, photinos $(\tilde{\gamma})$ and higgsinos $(\widetilde{h})^{\ddagger 2}$. Since the partners are not observed to be degenerate in mass with the normal particles, the supersymmetry must be badly broken (if it is a symmetry of nature at all). In general, if supersymmetry is relevant to understanding the weak scale, the masses of supersymmetric partners are expected to be of order $m_{\mathrm{W}}$, though some could be lighter.

To obtain the couplings of supersymmetric partners, one can take the electroweak and QCD couplings of the standard model, and replace the particles by their supersymmetric partners in pairs. Thus one can have transitions

$\mathrm{q} \rightarrow \mathrm{q} \gamma, \quad \tilde{\mathrm{q}} \rightarrow \mathrm{q} \tilde{\gamma}$,

with strengthe $\left(e^{2} / 4 \pi=\alpha\right)$,

${ }^{\ddagger 2}$ In general, one expects mixing between interaction eigenstates $\widetilde{w}^{ \pm}$and $\widetilde{\mathrm{h}}{ }^{ \pm}$and between $\widetilde{\gamma}, \widetilde{\mathrm{h}}^{0}$ and $\widetilde{\mathrm{z}}$; the actual phys ical mass eigenstates depend on model-dependent mixing angles. We shall continue to refer to the interaction eigenstates for simplicity of the discussion. See ref. [3] and references therein. These issucs are also discussed in refs. $[6$, 71 . 
$q \rightarrow q g, \quad \widetilde{q} \rightarrow q \widetilde{g}, \quad g \rightarrow \tilde{g} \widetilde{g}$,

with strength $g_{\mathrm{S}}\left(g_{\mathrm{s}}^{2} / 4 \pi=\alpha_{\mathrm{s}}=0.15\right)$, and

$\mathrm{W}^{ \pm} \rightarrow \mathrm{e}^{ \pm} \nu, \quad \widetilde{\mathrm{w}} \rightarrow \widetilde{\mathrm{e}}^{ \pm} \nu, \quad \widetilde{\mathrm{W}} \rightarrow \mathrm{e}^{ \pm} \widetilde{\nu}$,

with st rength $g_{2}\left(g_{2}=e / \sin \theta_{\mathrm{w}}\right)$, as well as many others. Additional vertices may occur, but those obtained by this procedure will always be present.

There are many ways to search for supersymmetric partners. Their signatures are rather definite. We will assume that the lightest supersymmetric partner is stable, and we take it to be the photino; our arguments here are not sensitive to this assumption. Since the photino interacts weakly with matter (by exchanging a heavy scalar quark or scalar lepton), it will escape a collider detector thereby resembling a neutrino. The decay modes of all other supersynmetric particles are very sensitive to their masses (as certain decay modes can be kinematically forbidden). For the purposes of this paper, we shall assume the following ordering for the masses: $\tilde{M}_{\gamma}<\tilde{M}_{\nu}<\widetilde{M}_{\mathrm{q}} \approx \tilde{M}_{\ell}$ $\approx \check{M}_{\mathrm{w}}<\tilde{M}_{\mathrm{g}}$. In this case, we would expect the following dominant decay modes: $\widetilde{\nu} \rightarrow \nu \widetilde{\gamma}[8], \widetilde{q} \rightarrow q \tilde{\gamma}$, $\widetilde{\ell} \rightarrow \ell \widetilde{\gamma}, \widetilde{\mathrm{w}} \rightarrow \ell \widetilde{\nu}$, and $\widetilde{\mathrm{g}} \rightarrow \overline{\mathrm{q}} \widetilde{\mathrm{q}} \rightarrow \mathrm{q} \overrightarrow{\mathrm{q}} \widetilde{\gamma}$. Note that one could have $\widetilde{M}_{\nu}<\tilde{M}_{\gamma} ;$ in both cases the scalar neutrino, like the photino, will escape the detector.

At a collider the supersymmetric partners will be produced in pairs. One expects $(\mathrm{q}, \overline{\mathrm{q}}, \mathrm{g}$ are quarks, antiquarks, and gluons in hadrons)

$\mathrm{q} \overline{\mathrm{q}} \rightarrow \widetilde{\mathrm{w}} \tilde{\mathrm{g}}, \tilde{\mathrm{w}} \widetilde{\mathbf{z}}, \tilde{\mathrm{w}} \tilde{\gamma}, \tilde{\mathrm{g}} \tilde{\gamma}, \ldots, \quad \mathrm{qg} \rightarrow \tilde{\mathrm{w}} \tilde{\mathrm{q}}, \tilde{\gamma} \tilde{\mathrm{q}}, \widetilde{\mathrm{g}} \tilde{\mathrm{q}}, \ldots$,

$\mathrm{gg} \rightarrow \widetilde{\mathrm{g}} \tilde{\mathrm{g}}, \tilde{\mathrm{q}} \tilde{\mathrm{q}}, \ldots$,

all to occur. Given a set of masses, the cross sections are all calculable $[9,10]$. When the final state particles decay, one will observe electrons and muons directly; $\mathrm{q}, \overline{\mathrm{q}}$, and $\mathrm{g}$ as jets; and $\nu, \tilde{\nu}, \tilde{\gamma}$ will escape. Thus, one might have

$\mathrm{qq} \rightarrow \tilde{\mathrm{w}} \tilde{\mathrm{g}} \rightarrow \mathrm{e}^{ \pm} \tilde{\nu} \mathrm{jj} \tilde{\gamma} \rightarrow \mathrm{e}^{ \pm} \mathrm{jj} \not_{\mathrm{T}}$,
$\mathrm{qg} \rightarrow \tilde{\mathrm{w}} \tilde{\mathrm{q}} \rightarrow \mathrm{e}^{ \pm} \tilde{\nu} \mathrm{j} \tilde{\gamma} \rightarrow \mathrm{e}^{ \pm} \mathrm{j} \not_{\mathrm{T}}$,

where $p_{\mathrm{T}}$ denotes significant missing $p_{\mathrm{T}}$ and $\mathrm{j}$ denotes a had ronic jet.

It is important that the missing $p_{\mathrm{T}}$ is made up of two particles, $\tilde{\nu}$ and $\tilde{\gamma}$. Their momenta can add, cancel, or anything in between. In particular, if the missing $p_{\mathrm{T}}$ is due to two or more particles, it would be incor-
T'able 1

This table lists some processes for the production of supersymmetric partners at a hadron collider. The constituent cross sections have been obtained by integrating eqs. (7) and (8) over all angles. Note that $s_{ \pm}=\sin \phi_{ \pm}$, where $\phi_{ \pm}$are modeldependent mixing angles. (See discussion below eqs. (7) and (8) to see how to obtain the $\mathrm{q} \overline{\mathrm{q}} \rightarrow \widetilde{\mathrm{g}} \tilde{\gamma}$ and $\mathrm{qg} \rightarrow \tilde{\mathrm{q}} \tilde{\gamma}$ cross sections.) For $\mathrm{qg} \rightarrow \widetilde{\mathrm{q}} \tilde{\mathrm{g}}$, we have used the results of ref. [9] (in particular, note the crratum to this referenoc). To get the numerical results above, we have chosen $\widetilde{M}_{\mathrm{q}}=\widetilde{M}_{\mathrm{W}}=40$ $\mathrm{GeV}, \widetilde{M}_{\mathrm{g}}=80 \mathrm{GeV}$ and $\widetilde{M}_{\gamma}=0$, and we have evaluated the cross sections at a $\sqrt{ } s$ given by the sum of the final state masses plus $30 \mathrm{GeV}$ (which roughly corresponds to where the cross section is a maximum). We have summed over all possible ele mentary processes involving valence quarks and over all appropriate possible final states. Presumably, experimental cuts and structure function effects reduce the cross sections given above by about a factor of five. The present data sample at the CERN collider corresponds to an integrated luminosity of $131 \mathrm{nb}^{-1}$ [5].

\begin{tabular}{|c|c|c|}
\hline $\begin{array}{l}\text { Constituent } \\
\text { process }\end{array}$ & Signature & $\begin{array}{l}\text { Constituent } \\
\text { cross section (nb) }\end{array}$ \\
\hline $\mathrm{q} \overline{\mathrm{q}} \rightarrow \widetilde{\mathrm{w}}^{ \pm} \widetilde{\mathrm{g}}$ & $\begin{array}{l}Q \pm \mathrm{jj} P \mathrm{~T} \\
\ell \pm \mathrm{jj}\end{array}$ & $0.11\left(s_{+}^{2}+s_{-}^{2}\right)+0.16 s_{+} s_{-}$ \\
\hline $\mathrm{qg} \rightarrow \widetilde{\mathrm{w}}^{ \pm} \widetilde{\mathrm{q}}$ & $\begin{array}{l}\ell^{ \pm} \mathrm{j} R T \\
\ell^{ \pm} \mathrm{j}\end{array}$ & $0.03\left(s_{-}^{2}+s_{+}^{2}\right)$ \\
\hline $\mathrm{qg} \rightarrow \widetilde{\mathrm{q}} \widetilde{\mathrm{g}}$ & jjjpx & 3.83 \\
\hline $\mathrm{q} \overline{\mathrm{q}} \rightarrow \tilde{\mathrm{g}} \tilde{\gamma}$ & $\mathrm{jj} p \mathrm{~T}$ & 0.06 \\
\hline $\mathrm{qg} \rightarrow \widetilde{\mathrm{q}} \tilde{\gamma}$ & $\mathrm{j} p \mathrm{~T}$ & 0.014 \\
\hline
\end{tabular}

rect to attribute to it a momentum four-vector whose square is zero (as is appropriate for a neutrino). Thus a clear prediction if such events occur and are interpreted as in eqs. (5) and (6) is that there should be a continuum of such events from large missing $p_{1}$ down to no missing $p_{\mathrm{T}}$ (the latter give events that could be interpreted as violating lepton number).

In table 1 we give a set of reactions, the associated signatures, and numerical results for the constituent cross sections. These numbers have been obtained as follows. First, we have computed the elementary cross sections for reactions (5) and (6). Our results are:

$$
\begin{aligned}
& \mathrm{d} \sigma(\mathrm{u} \overline{\mathrm{d}} \rightarrow \widetilde{\mathrm{g}} \widetilde{\mathrm{w}}) / \mathrm{d} t=\left(2 \pi \alpha \alpha_{\mathrm{s}} / 9 s^{2} \sin ^{2} \theta_{\mathrm{w}}\right) \\
& \quad \times\left(\frac{\sin ^{2} \phi_{-}\left(\widetilde{M}_{\mathrm{w}}^{2}-t\right)\left(\widetilde{M}_{\mathrm{g}}^{2}-t\right)}{\left(\tilde{M}_{\mathrm{q}}^{2}-t\right)^{2}}\right. \\
& \left.\quad+\frac{\sin ^{2} \phi_{+}\left(\tilde{M}_{\mathrm{w}}^{2}-u\right)\left(\tilde{M}_{\mathrm{g}}^{2}-u\right)}{\left(\widetilde{M}_{\mathrm{q}}^{2}-u\right)^{2}}+\frac{2 s \widetilde{M}_{\mathrm{w}} \widetilde{M}_{\mathrm{g}} \sin \phi_{+} \sin \phi_{-}}{\left(\widetilde{M}_{\mathrm{q}}^{2}-t\right)\left(\widetilde{M}_{\mathrm{q}}^{2}-u\right)}\right),
\end{aligned}
$$




$$
\begin{gathered}
\mathrm{d} \sigma\left(\mathrm{ug} \rightarrow \tilde{\mathrm{d}}_{\mathrm{L}} \widetilde{\mathrm{w}}\right) / \mathrm{d} t=\left(\pi \alpha \alpha_{\mathrm{s}} \sin ^{2} \phi_{-} / 12 s^{2} \sin ^{2} \theta_{\mathrm{w}}\right) \\
\times\left(\frac{\tilde{M}_{\mathrm{w}}^{2}-u}{s}-\frac{2 \tilde{M}_{\mathrm{q}}^{2}\left(\tilde{M}_{\mathrm{w}}^{2}-t\right)}{\left(\widetilde{M}_{\mathrm{q}}^{2}-t\right)^{2}}\right. \\
\left.+\frac{2 s \tilde{M}_{\mathrm{w}}^{2}+2\left(t \quad \widetilde{M}_{\mathrm{w}}^{2}\right)\left(\tilde{M}_{\mathrm{w}}^{2}-\tilde{M}_{\mathrm{q}}^{2}\right)}{s\left(\tilde{M}_{\mathrm{q}}^{2}-t\right)}\right)
\end{gathered}
$$

where

$$
\begin{aligned}
t= & -\frac{1}{2}\left(s-\widetilde{M}_{\mathrm{w}}^{2}-\widetilde{M}^{2}\right)+\frac{1}{2}\left[\left(s-\widetilde{M}_{\mathrm{w}}^{2}-\widetilde{M}^{2}\right)^{2}\right. \\
& \left.-4 \widetilde{M}_{\mathrm{W}}^{2} \tilde{M}^{2}\right]^{1 / 2} \cos \theta,
\end{aligned}
$$

$\tilde{M}$ is the gluino or scalar quark mass in eqs. (7) and (8) respectively and $\theta$ is the COM scattering angle of the produced $\widetilde{w}$. The symbol $\widetilde{w}$ above signifies the lighter of two mass-eigenstates which arise from charged wino-higgsino mixing. The mixing is in general parameterized by two mixing angles $\phi_{ \pm}$. The $\widetilde{w}^{+} \widetilde{\mathrm{u}}_{\mathrm{L}} \mathrm{d}$ vertex is proportional to $\sin \phi_{+}$, and the $\widetilde{w}^{+} \widetilde{d}_{L} u$ vertex is proportional to $-\sin \phi_{-}$. (See ref. [3] for further discussion on the mixing. The cross sections for the production of the heavier of the two masseigenstates can be obtained from eqs. (7) and (8) by the replacement $\sin \phi_{ \pm} \rightarrow \pm \cos \phi_{ \pm}$.) Differential cross sections for $\mathrm{q} \overline{\mathrm{q}} \rightarrow \tilde{\mathrm{g}} \tilde{\gamma}$ and $\mathrm{qg} \rightarrow \widetilde{\mathrm{q}} \tilde{\gamma}$ are obtained from eqs. (7) and (8) by the replacement $\pm g_{2} \sin \phi_{ \pm} \rightarrow$ $e e_{\mathrm{q}} \sqrt{ } 2$ where $e_{\mathrm{q}}$ is the quark charge in units of $e$. For simplicity, we assume that the scalar quarks $\tilde{q}_{L}$ and $\tilde{\mathrm{q}}_{R}$ are degenerate in mass. Then there is no interference between $\widetilde{\mathrm{q}}_{L}$ and $\widetilde{\mathrm{q}}_{R}$ exchange so that we may simply add cross sections, resulting in an additional factor of 2. [In contrast, only $\tilde{\mathrm{q}}_{L}$ is involved in the reactions (5) and (6) since $\widetilde{q}_{R}$ does not couple to $\widetilde{w}$.] Cross sections for $\mathrm{qg} \rightarrow \widetilde{\mathrm{g}} \widetilde{\mathrm{q}}$ are given in refs. $[9,10]^{\neq 3}$. We note that perturbative calculations usually underestimate the production cross sections. However, our numerical results below are based solely on the perturbative cross sections given above.

Events of the type $\mathrm{e}^{ \pm} \mathrm{jj} \mathbb{R}_{\mathrm{T}}$ and $\mathrm{e}^{ \pm} \mathrm{j} \mathbb{p}_{\mathrm{T}}$ have been reported recently by the UA2 collaboration [5] at the CERN pp collider. Events of the kind $j p_{\mathrm{T}}$ have been reported by the UA1 group as well [11]. It is clear that such events are a natural prediction of supersymmetry. For gluino masses in the range $70-100 \mathrm{GeV}$, scalar-quark masses $40-60 \mathrm{GeV}$, wino masses $35-50$

\footnotetext{
\$3 In ref. [9] (erratum), there is a misprint in $\sigma$ (qg $\rightarrow \widetilde{\mathrm{q}} \widetilde{\mathrm{g}}$ ): a factor $\Delta / 9$ should read $\Delta /(9 s)$.
}

$\mathrm{GeV}$, and $\tilde{M}_{\mathrm{q}}>\tilde{M}_{\mathrm{w}}>\tilde{M}_{\nu}$, the cross sections and kinematic properties of these events appear to be consistent with the predictions of supersymmetry. A detailed analysis is now being carried out to confirm this claim [12].

The UA2 candidate events [5] have appeared in a run with an integrated luminosity of $1.3 \times 10^{35} / \mathrm{cm}^{2}$. The constituent cross section for $\mathrm{q} \overline{\mathrm{q}} \rightarrow \widetilde{\mathrm{w}} \widetilde{\mathrm{g}}$ at $\sqrt{s}=$ $150 \mathrm{GeV}$ is about $1.2 \times 10^{-34} \mathrm{~cm}^{2}$ (where we have taken $\left.\sin \phi_{ \pm}=1\right)$ including a factor of $1 / 3$ for the branching ratio for $\widetilde{\mathrm{w}} \rightarrow \mathrm{e} \widetilde{\nu}$, giving about 16 basic events possible. Experimental cuts and structure function effects will reduce the rate by about another factor of 5 , giving an expected rate of a few jjj $_{\mathrm{T}}$ events. Production of $\tilde{w} \hat{q}$ is somewhat smaller, giving about one event of $\ell_{j} p_{T}$ by similar reasoning.

Before any anomalous events can be interpreted as new physics, it is crucial to thoroughly examine all standard model processes and backgrounds which can produce such events. If these events are to be interpreted as the observation of supersymmetry, they must of course be confirmed by other detectors and by increased statistics in future runs. We can also give some predictions that must be verified. The predictions either come from rearranging the particles in an "observed" decay, or by arranging which particles are pair-produced. Thus, if $\mathrm{q} \overline{\mathrm{q}} \rightarrow \widetilde{\mathrm{w}} \tilde{\mathrm{g}} \rightarrow \mathrm{e}^{ \pm} \mathrm{jj} \tilde{\nu} \tilde{\gamma}$ and the $\tilde{\nu} \tilde{\gamma}$ are combined to give missing $p_{\mathrm{T}}$ in some events, they will point oppositely to give little missing $\gtrless_{T}$ in other events. One also produces $\mathrm{qg} \rightarrow \widetilde{\mathrm{g}} \tilde{\mathrm{q}} \rightarrow \mathrm{jjj} p_{\mathrm{X}}$; i.e. events with three jets and missing $p_{\mathrm{T}}$ Inust be observed. The rate as shown in table 1 is very large. If such events are not observed, one would conclude that either $\widetilde{M}_{\mathrm{q}}>\widetilde{M}_{\mathrm{g}}$ (in which case $\widetilde{\mathrm{q}} \rightarrow \mathrm{q} \widetilde{\mathrm{g}}$, implying a more complicated signature), or the $\widetilde{\mathrm{g}}$ and $\widetilde{\mathrm{q}}$ masses are much heavier than we have assumed here. In either case, the scenario presented in this paper would be incorrect. In addition, we expect $\mathrm{q} \overline{\mathrm{q}} \rightarrow \tilde{\mathrm{g}} \tilde{\gamma}$ and $\mathrm{qg} \rightarrow \tilde{\mathrm{q}} \tilde{\gamma}$ to produce the events $\mathrm{jj} \mathbb{R}_{\mathrm{T}}$ and $\mathrm{j} \boldsymbol{p}_{\mathrm{T}}$ respectively, as indicated on table 1 . The latter signature is quite spectacular; standard model backgrounds (such as pp $\rightarrow$ $\mathrm{Z}^{0}+\mathrm{g}, \mathrm{Z}^{0} \rightarrow \nu \bar{\nu}$ and $\mathrm{p} \overline{\mathrm{p}} \rightarrow \mathrm{W}^{ \pm} \rightarrow \tau^{ \pm} \nu, \tau^{ \pm} \rightarrow \nu+$ hadrons) are in principle known and can be subtracted.

It is important to realize that if the anomalous events are interpreted as the production of supersyrilmetric partners, then both signatures and sizes of the cross sections of various related processes described above must come out right. Thus, one will be able to 
soon rule out a supersymmetric explanation for these events if it is incorrect.

Further consequences of the scenario presented here are that there should be supersymmetric decays of the $\mathrm{W}$; either $\mathrm{W} \rightarrow \tilde{\ell} \widetilde{\nu}[13]$ or $\mathrm{W} \rightarrow \widetilde{\mathrm{w}} \tilde{\gamma}$ [6]. With our assumption on the various masses, these events would be very similar [14] consisting of a final state of $\ell^{ \pm} p_{T}$. The leptons would tend to emerge at a smaller $p_{\mathrm{T}}$ as compared to $\mathrm{W} \rightarrow \ell \nu$ events; a larger sample of such events would be needed before an anomalous signal could be inferred. Note that the $\tilde{w} \tilde{\gamma}$ final state could also be produced via continuum $\mathrm{q} \overline{\mathrm{q}}$ $\rightarrow \tilde{w} \tilde{\gamma}[7]$ with a signal similar to the one just discussed. In addition, one can search for evidence of supersymmetry at other accelerators. For example, if photinos and/or scalar-neutrinos are rather light (as we have assumed here), then they could be indirectly inferred from "neut rino-counting" experiments $\left(\mathrm{e}^{+} \mathrm{e}^{-} \rightarrow \gamma+\right.$ nothing seen), where the undetected particles produced could be $\tilde{\gamma} \tilde{\gamma}[15]$ or $\tilde{\nu} \widetilde{\nu}$ (in addition to the expected $\nu \bar{\nu})$. Such experiments are now in progress at PEP [16].

We have not discussed here the production of the $\tilde{z}$. Examples include final states consisting of $\widetilde{z} \widetilde{\mathrm{W}}, \widetilde{z} \widetilde{\gamma}, \widetilde{z} \widetilde{\mathrm{g}}$, $\widetilde{z} \widetilde{q}$ and $\tilde{z} \widetilde{z}$. One typically finds reduced cross sections due to smaller neutral current couplings [10]. It is important to emphasize that the number of events expected of a particular signature (such as those given in table 1) depends very sensitively on the masses chosen for the supersymmetric partners. For example, if the $\widetilde{\mathrm{w}}$ were sufficiently heavier than the scalar quark, then the dominant $\tilde{\mathrm{w}}$ decays would be $\widetilde{\mathrm{w}} \rightarrow \mathrm{q} \tilde{\mathrm{q}}$ resulting in no final states involving leptons. Of course, we chose one particular ordering of the supersymmetric masses inspired by the results of UA2 [5]. This is certainly sensible in that it will be the experiments which will ultimately decide whether supersymmetry is viable and which masses are indicated. On the other hand, supersymmetry cannot explain an arbitrary non-standard event. For example, supersymmetry does not normally lead to events with isolated hard photons. Furthermore, one can never directly reconstruct a supersymmetric partner via an invariant mass plot as some energy is always lost to an escaping photino. Thus, there is no supersymmetric explanation for the observed ee $\gamma$ events at the CERN collider [17].

In conclusion, the CERN $\bar{p}$ collider is a valuable tool to search for supersymmetric phenomena. In general, if supersymmetric particles are accessible at $\sqrt{s}=540 \mathrm{GeV}$, one expects to see a general class of anomalous looking events. These events have substantial missing $p_{\mathrm{T}}$, one or more hadronic jets and possibly an isolated electron or muon. The exact number of events of each kind is highly model dependent. We have proposed one general scenario which could lead to events similar to ones now being reported at the CERN p $\bar{p}$ collider. We look forward with eager anticipation to future running at the CERN collider to see whether the evidence for new physics will indeed emerge.

Conversations with R. Battiston, D. Cline, C. Conta, P. Darriulat, P. Hansen and L. Littenberg are gratefully acknowledged.

\section{References}

[1] P. Fayet and S. Ferrara, Phys. Rep. 32C (1977) 249; A. Salam and J. Strathdee, Fortschr. Phys. 26 (1978) 57; J. Wess and J. Bagger, in: Supersy mmetry and supergravity (Princeton U.P., Princeton, NJ, 1983);

J. Gates, M. Grisaru, M. Rocek and W. Siegel, in: Superspace or 1001 lectures in supersy mmetry (Benjamin, Reading, MA, 1983).

[2] C.A. Savoy, in: Beyond the standard model, Proc. 18th Rencontre de Moriond (1983), ed. J. Tran Thanh Van (Editions Fruntieres, Dreux, 1984); P. Nath, R. Arnowitt and A.H. Chamseddine, Northeastern preprint NUB\#2613; lectures 1983 Summer Workshop on Particle physics (Trieste).

[3] H.E. Haber and G.L. Kane, University of Michigan preprint UM HE TH 83-17 (1984), to be published in Phys. Rep.

[4] I. Hinchliffe and L. Littenberg, in: Proc. 1982 DPF Summer Study of Elementary particle physics and future facilities, eds. R. Donaldson, R. Gustafson and F. Paige (American Physical Society, New York, 1982) p. 242; P. Fayet, in: Proc. 21 st Intern. Conf. on High energy physics (Paris), eds. P. Petiav and M. Porneuf (Editions de Physique, Paris, 1982) p. C3673;

D.V. Nanopoulos, A. Savoy-Navarro and Ch. Tao, Proc. Supersymmetry versus experiment workshop, Phys. Rep. 105 (1982) 1;

J. Ellis, Proc. 11th SLAC Summer Institute on Particle physics, ed. P. McDonough, SLAC report no.267, p. 239.

[5] P. Hansen, talk Erice Flavor Workshop (March 1984); M. Livan, talk Rencontre de Moriond (March 1984); A. Roussarie, talk Berne Collider Meeting (March 1984); UA2 Collab., P. Bagnaia et al., Phys. Lett. 139B (1984) 105 . 
[6] S. Weinberg, Phys. Kev. Lett. 50 (1983) 387; A.H. Chamseddine, R. Arnowitt and P. Nath, Phys. Rev. Lett. 49 (1982) 970; Phys. Lett. 129B (1983) 445; J.M. Frere and G.L. Kane, Nucl. Phys. B223 (1983) 331; P. Fayet, Phys. Lett. 125B (1983) 178; 133 B (1983) 363; J. Ellis, J. Hagelin, D.V. Nanopoulos and M. Srednicki, Phys. Lett. 127 B (1983) 233;

D.A. Dicus, S. Nandi and X. Tata, Phys. Lett. 129B (1983) 451;

B. Grinstein, J. Polchinski and M.B. Wise, Phys. Lett. 130B (1983) 285;

V. Barger, R.W. Robinett, W.Y. Keung and R.J.N. Phillips, Phys. Rev. D28 (1983) 2912;

D.A. Dicus, S. Nandi, W.W. Repko and X. Tata, Phys. Rev. D29 (1984) 67.

[7] V. Barger, R.W. Robinett, W.Y. Keung and R.J.N. Phillips, Phys. Lett. 131B (1983) 372.

[8] R.M. Barnett, H.E. Haber and K.S. Lackner, Phys. Lett. $126 B$ (1982) 64; SLAC-PUB-3224, to be published in Phys. Rev. D.

[9] P.R. Harrison and C.H. Llewellyn Smith, Nucl. Phys. B213 (1983) 223; B223 (1983) 542 (E).
[10] S. Dawson, E. Eichten and C. Quigg, FERMILAB-PUB83/82-THY (1984), in preparation; E. Eichten, I. Hinchliffe, K. Lane and C. Quigg, FERMILAB-PUB-84/17-THY (1984), in preparation .

[11] UA1 Collab., G. Arnison et al., Phys. Lett. 139B (1984) 115.

[12] R.M. Barnett, H.E. Haber and G.L. Kane, in preparation.

[13] R.M. Barnett, H.E. Haber and K.S. Lackner, Phys. Rev. Lett 51 (1983) 176; SLAC-PUB-3225, to be published in Phys. Rev. D; R. Barbieri, N. Cabibbo, L. Maiani and S. Petrarca, Phys. Lett. 127B (1983) 458.

[14] R.M. Barnett and H.E. Haber, preprint, in preparation.

[15] P. Fayet, Phys. Lett. 117B (1982) 460; J. Ellis and J. Hagelin, Phys. Lett. 122B (1982) 303.

[16] D. Burke et al., SLAC experiment, in progress; MAC Collab., SLAC experiment, in progress.

[17] UAl Collab., G. Arnison et al., Phys. Lett. 126B (1983) 398

UA2 Collab., P. Bagnaia et al., Phys. Lett. 129B (1983) 130 . 